\title{
Pecios italianos localizados en el medio terrestre. El caso del pecio tardo-romano de Rávena
}

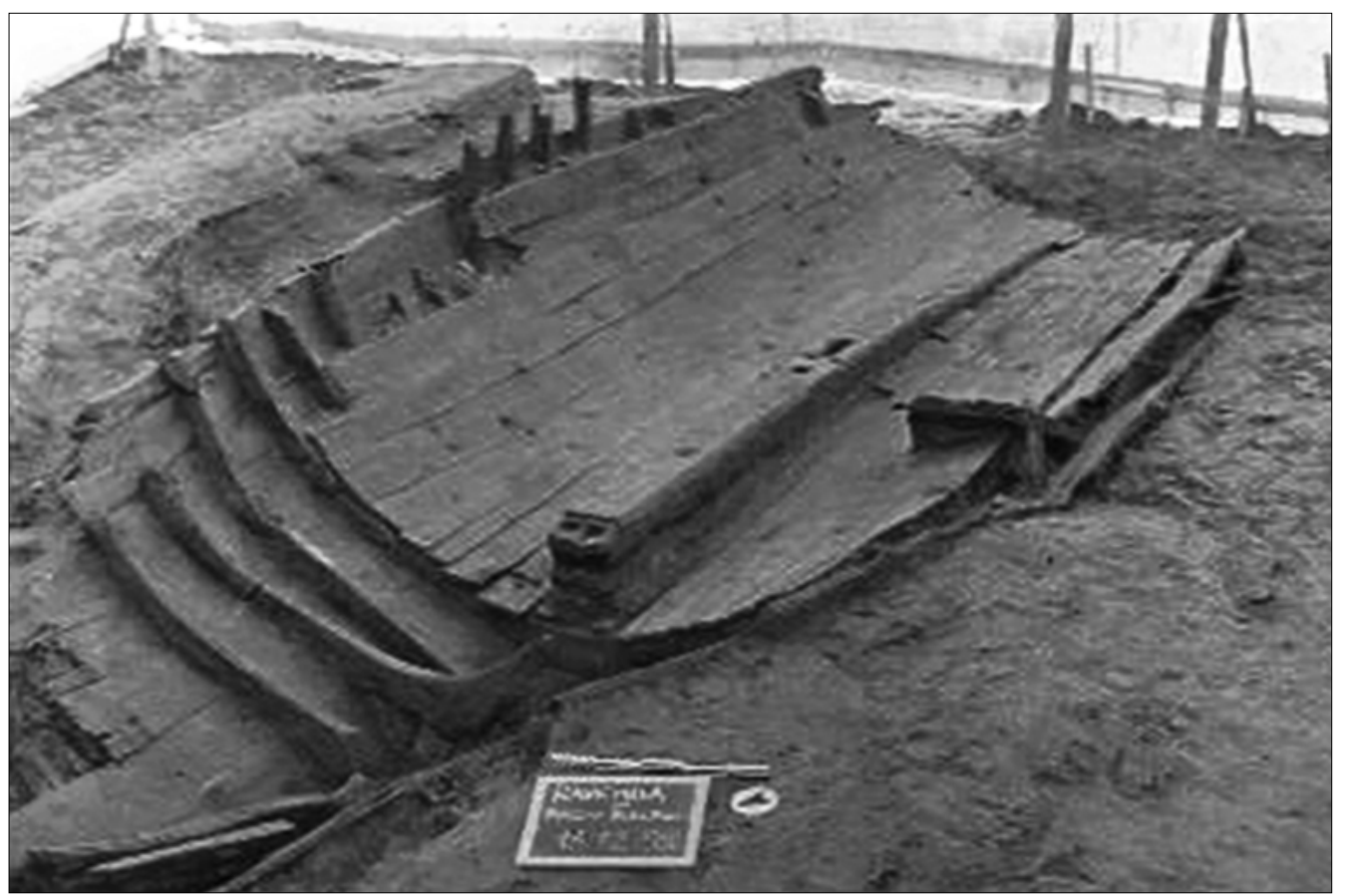

Traducido del italiano por Lourdes Márquez Carmona

\section{Stefano Medas}

Istituto Italiano di Archeologia e Etnologia

Navale, Venecia

\section{Resumen}

Como consecuencia de la dinámica litoral, encargada de la movilización de los aportes sedimentarios, y de la actividad neotectónica, causante la elevación o hundimiento de determinadas zonas costera a lo largo de los siglos, el trazado de la línea de costa han cambiado considerablemente respecto a la antigüedad. Muchos estuarios fluviales o lagunas litorales han sufrido por ellos procesos de relleno sedimentario, cubriendo antiguas zonas portuarias o de fondeo. Es frecuente en la actualidad que, durante la ejecución de obras públicas, en estas antiguas zonas costeras hoy desecadas, se pongan al descubierto de restos de viejas naves en inmejorable estado de conservación; naves que conforman una valiosa información para los arqueólogos y la arqueología naval.

\section{Palabras clave:}

Arqueología naval/ pecios romanos/ cambios costeros/ arquitectura naval/ arqueología subacuática. 
0
0
$+\pi$
2
0
0
0
2
0
0
0
0
0
0
0
2
2
0
1
$<$
Generalmente el descubrimiento de los restos de una nave antigua se produce en un contexto arqueológico subacuático. Sin embargo, existen casos célebres de descubrimientos acontecidos en tierra firme, en yacimientos terrestres o húmedos, que incluyen también áreas que, si en un principio se encontraban sumergidas, en la actualidad se hallan desecadas '. Ello se debe a que la situación geomorfológica de muchas zonas costeras ha cambiado notablemente respecto a la antigüedad, sobre todo en el caso de aquellos territorios situados a cotas bajas respecto al mar y próximos a desembocaduras fluviales. Tales procesos de aterramiento son debidos al avance de la línea de costa producidos por el transporte de sedimentos fluviales y en ciertas áreas, también al fenómeno de subsidencia, que ha determinado la colmatación de superficies anteriormente ocupadas por el agua. Son particularmente activos los fenómenos en las zonas lagunares $^{2}$. En zonas interiores pertenecientes a cuencas fluviales o lacustres, sobre todo en llanura, se han producido casos similares provocados, bien por acontecimientos naturales como los aluviones, provocando el cambio de curso de ríos o la desecación de áreas pantanosas o lacustres, o bien por la intervención del hombre, como en el caso de los trabajos de canalizaciones o de drenaje de terrenos.

Por estos motivos, la superficie de agua de algunas áreas portuarias o de simples zonas de atraques ha sido ocupada completamente por tierra firme, a menudo junto a embarcaciones abandonadas por desuso o bien por sucesos desastrosos o imprevistos (basta recordar el puerto de Claudio en Ostia, o los puertos de Marsella, de Rávena, de Pisa). Por ello, la arqueología naval tiene como campo de actuación no sólo el contexto subacuático, sino también el terrestre.

Antes de pasar al tema específico que nos ocupa, creemos que es útil incluir una breve nota de carácter terminológico y disciplinar, importante, dada la confusión que a veces se crea, sobre todo en ámbitos divulgativos, en cuanto a la definición del término "ar-

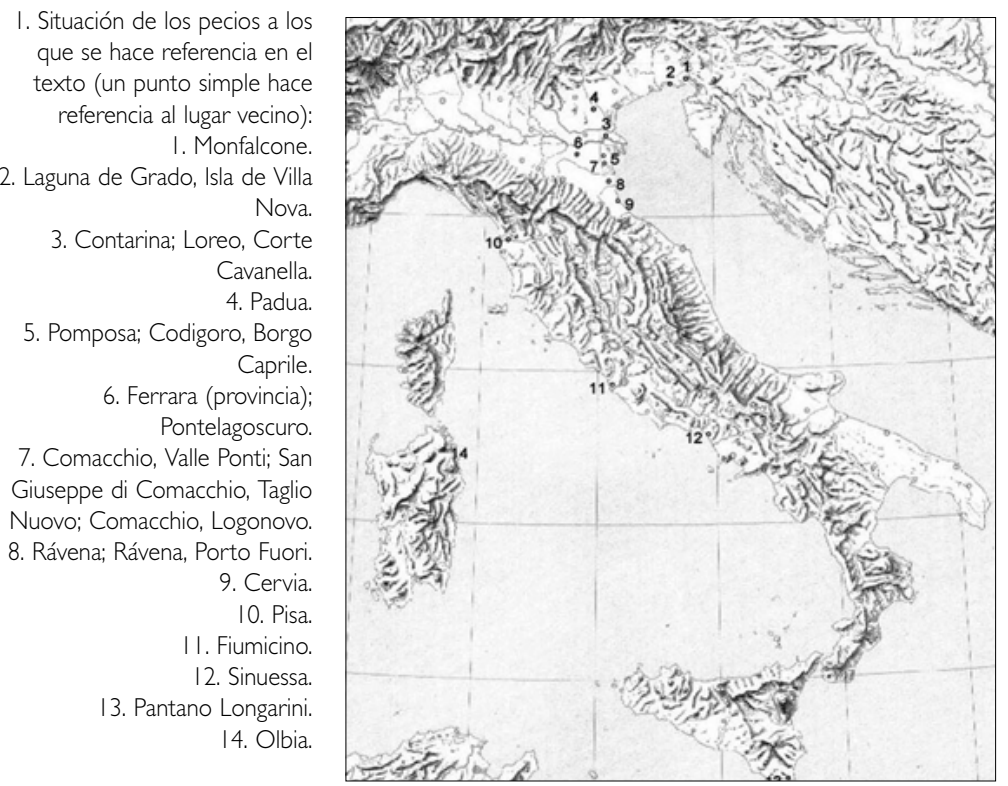

queología" 3 . Si la arqueología subacuática debe entenderse como "arqueología debajo del agua", respondiendo a una técnica operativa específica desarrollada únicamente en el contexto ambiental en el cual se trabaja, la arqueología del agua comprendería las evidencias relativas a los más diversos contextos de relación hombre-agua en el tiempo, ya sea en las condiciones originarias o en evoluciones sucesivas, así como las circunstancias operativas a las que se enfrenta actualmente el arqueólogo. La arqueología del agua abarcaría entonces, la investigación tanto en contexto subacuático como en húmedos o desecados ${ }^{4}$. Por su parte, la arqueología naval es una disciplina autónoma que tiene por objeto de estudio las embarcaciones, sus aspectos técnicos y culturales, valiéndose actualmente tanto de la arqueología subacuática como de la arqueología terrestre (en el caso de restos materiales que han sufrido un proceso de aterramiento o cubrimiento). Al igual que la arqueología del agua, la arqueología naval tiene una amplia tradición investigadora, desarrollándose notablemente desde la mitad del siglo XIX, siendo pues antecedente de la arqueología subacuática.

La investigación se sirvió durante mucho tiempo de fuentes iconográficas y literarias exclusivamente, salvo raras excepciones en las que se produjeron hallazgos en el medio terrestre. La recuperación de la gigantesca nave del lago de Nemi realizada entre los años 1928 y 1932 con la desecación parcial del lago, puso de manifiesto con gran fuerza cual podía ser la potencialidad ofrecida por la investigación arqueológica en un contexto subacuático; aunque si en este caso no se puede hablar todavía de arqueología subacuática como la entendemos hoy, sí está considerado como una actuación arqueológica excepcional que, por primera vez, ha trasladado fuera del agua las naves antiguas, ofreciendo la posibilidad de un conocimiento directo. El desarrollo radical se produjo en efecto, con la aparición de la arqueología subacuática, es decir, con la intervención directa de los arqueólogos debajo del agua a partir de los años cincuenta y sobre todo de los años sesenta. Esta nueva disciplina se convirtió en un aliado fundamental de la arqueología naval, ofreciendo la posibilidad de una aproximación directa a los restos que yacían en el fondo del mar, lagos, lagunas o ríos 5 .

A continuación presentamos una sintética reseña de los principales hallazgos italianos. Desgraciadamente muchos de ellos, pertenecientes a antiguos descubrimientos, se han perdido o se han deteriorado irreparablemente a causa del proceso de desecación sufrido por la madera, por no ser sometidos con prontitud a un tratamiento de conservación adecuado.

La zona del delta del Po se presenta particularmente rica en descubrimientos, ocurridos casualmente durante las labores de drenaje de áreas pantanosas y lagunares, durante la excavación de canales o de canteras de grava o arcilla. Remontándonos al año 1898 , durante los trabajos de excavación de un canal, se produjo el descubrimiento de dos hallazgos enterrados en Contarina (Rovigo), datados respectivamente 
en torno al I 300 y a la mitad del s. XVI. De estos importantes hallazgos y de las sucesivas intervenciones de excavación, se conserva un detallado estudio publicado en el año 1900, ilustrado con documentación gráfica de los dos yacimientos ${ }^{6}$.

A este primer descubrimiento se añadieron muchos otros (figura I). Todos sucedieron de modo casual, con ocasión del drenaje de áreas pantanosas o lagunares, o durante trabajos de excavación en canteras de grava o arcilla, en otras ocasiones debido a la apertura de canales, o bien por excavaciones realizadas para construir los cimientos de edificios o para la instalación de tuberías, etc. La naturaleza fortuita de estos hallazgos, destrozados en numerosas ocasiones por maquinas, supuso la pérdida en muchos casos del contexto originario del yacimiento y de materiales susceptibles de ser datados, reduciéndose a pocos elementos inconexos y descontextualizados; sólo en contadas ocasiones fueron objeto de una intervención arqueológica, por lo que resulta generalmente difícil una definición cronológica precisa de los mismos. Por tanto, el número de hallazgos es ciertamente mucho mayor respecto a lo que ha podido ser efectivamente constatado, y sobre todo, respecto a lo que ha sido destruido sin que la autoridad competente en la tutela del patrimonio arqueológico hubiese tenido conocimiento ${ }^{7}$.

El período comprendido entre el otoño de 1998 y el verano de 1999 representa un momento excepcional y único para la historia de la arqueología naval italiana. En el transcurso de pocos meses, durante la realización de trabajos públicos de diversa naturaleza, se descubrieron una gran cantidad de restos en tierra, en Rávena, Pisa y Olbia. Debido a la naturaleza de estos restos, se iniciaron rápidamente las actuaciones arqueológicas que todavía están desarrollándose en Pisa y Olbia.

Tales descubrimientos evidencian cuatro aspectos fundamentales: en primer lugar la gran oportunidad ofrecida por la aparición de restos en un contexto terrestre, con la posibilidad de excavar y estudiar los materiales y la estructura de los barcos según los sistemas de la arqueología terrestre, sin los condicionamientos y las limitaciones de carácter técnico que intervienen en yacimientos subacuáticos. En segundo lugar, hay que señalar que, en este tipo de yacimientos, el estado de conservación y la variedad tipológica de las embarcaciones son verdaderamente excepcionales. En tercer lugar, se hace posible un análisis extenso del contexto ambiental en los cuales se hallan los restos ya sea de tipo natural o representado por estructuras portuarias. Por último, en cuarto lugar, surgen dificultades de carácter arqueológico y técnico en excavaciones complejas, como es el caso de Pisa, y la necesidad de planificar un inmediato programa de conservación y de depósito de los restos materiales en los museos, lo cual supone naturalmente compromisos técnicos y financieros, bien sea a corto o a medio plazo, o bien sea a largo plazo. En cada caso, durante las diversas fases de intervención, se deben adoptar estrategias específicas, que supongan la adopción de soluciones innovadoras, además de experimentales.
La relación de los principales hallazgos atendiendo a una división regional es la siguiente:

\section{Friuli Venecia Giulia}

- Monfalcone (Gorizia): durante la excavación de una villa rústica romana en la localidad de Lisert, en el año 1972, fueron descubiertos los restos de una embarcación romana datada entre los siglos I y |II d.C. Después de las labores de recuperación, se conservaron durante algunos años sumergidos en un depósito construido expresamente; seguidamente se desarrollaron las labores de consolidación de la madera. Actualmente se hayan expuestos en el Museo Arqueológico Nacional de Aquileia ${ }^{8}$.

- Isla de Villa Nova, Laguna di Grado (Gorizia): durante los trabajos de excavación para la apertura de un pequeño embarcadero, fueron extraídos del fondo lagunar algunos restos de una nave romana (probablemente del s. I d. C.), entre los cuales apareció un fragmento de quilla de gran longitud, que conservaba aún parte de la roda?

\section{Veneto}

- Loreo, localidad de Corte Cavanella (Rovigo): en los años 1983 y 1985 respectivamente fueron descubiertas dos pequeñas embarcaciones datadas entre finales del siglo I y comienzos del s. II, en el área de un asentamiento romano caracterizado por la presencia de algunos canales navegables. El primero hallado in situ, yacía sobre un derrumbe de restos de tegulas y vasijas que originalmente constituían el cubrimiento de una dársena cerrada. El segundo había sido parcialmente desmontado en tiempos antiguos y reutilizado como un puente de paso.

- Padua; fueron descubiertos restos de una embarcación romana (parte de la tablazón), datada entre el siglo I e inicios del s. II d. C., que habían sido parcialmente reutilizados en los trabajos de drenajes en relación probablemente de una estructura portuaria en la ribera de un paleocauce del río Brenta.

- Oderzo (Treviso): estructura de tablas verticales "cosidas"; hay que descartar que haya sido realizada reutilizando también parte del casco de la embarcación ${ }^{10}$.

\section{Emilia Romagna}

- Pomposa (Ferrara), Bosco Spada: en 1922 fueron descubiertos los restos de una embarcación probablemente de cronología alto-medieval, que se destruyeron rápidamente.

- Pontelagoscuro (Ferrara): en 1953 se encontraron cerca del dique del río Po grandes tablazones de madera de encina aún ensambladas, que probablemente pertenecían a una barcaza utilizada como molino flotante, también de época alto-medieval. 


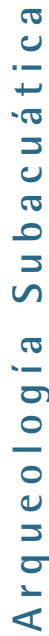

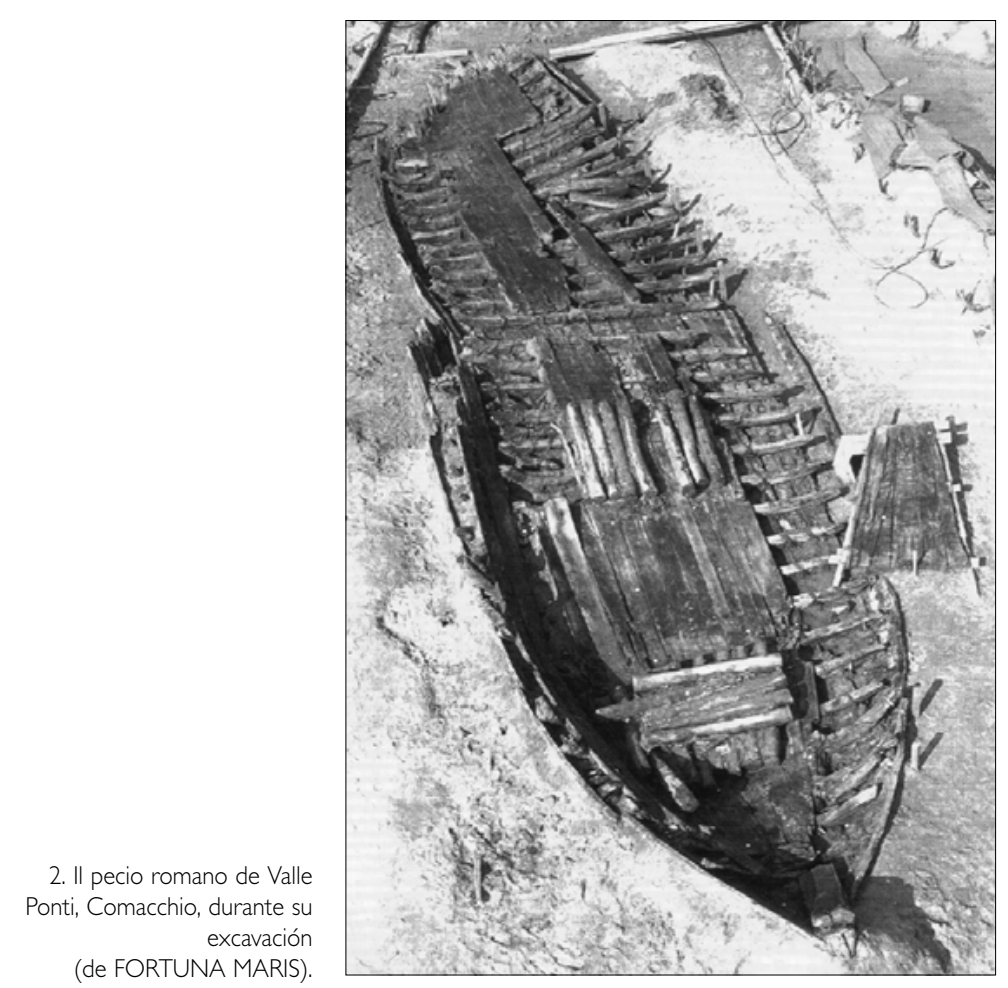

(de FORTUNA MARIS).

- Borgo Caprile, Codigoro (Ferrara); los restos de una embarcación "cosida" alto-medieval fueron descubiertos en el año 1956, dejándose in situ.

- Cerca del canal Logonovo, Comacchio (Ferrara): los restos de una nave tardo-medieval fueron recuperados en el año 1959.

- En varias localidades de la zona del delta, en la provincia de Ferrara, entre finales del 1800 y mediados del siglo XX fueron halladas numerosas embarcaciones monóxilas casi todas pertenecientes a época tardo-romanal".

- Cervia (Rávena): en una cantera de grava fueron recuperados en el año 1956 restos de una embarcación "cosida" tardo-romana 12.

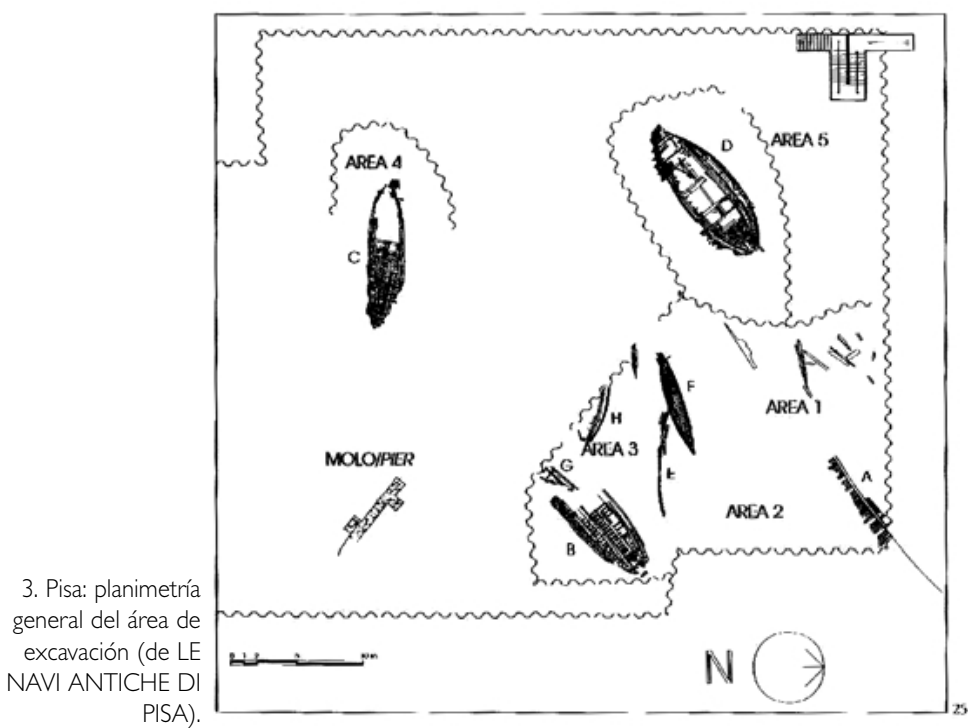

- Localidad de Porto Fuori (Rávena): durante unos trabajos de extracción de arena en el año 1979 salieron a la luz restos de una embarcación medieval, datadas entorno al año 1300 13.

- Valle Ponti, Comacchio (Ferrara): este descubrimiento excepcional, que se encuentra entre los más importantes de la arqueología naval italiana, sucedió durante los trabajos de dragado de un canal en otoño de 1980, cuando se localizaron restos de cronología romana datados a fines del s. I a.C. (figura 2). Las excavaciones iniciadas posteriormente condujeron a la recuperación de los mismos durante el período invernal de 1988-1989 y el inicio del tratamiento de conservación. Actualmente se encuentran en Comacchio, en la sección del museo creada expresamente para la exposición de la nave romana. Los estudios de los sedimentos del yacimiento han permitido establecer que la nave naufragó cerca de la playa, al tocar fondo en un banco de arena cerca de una desembocadura fluvial. Además de buena parte del casco, de gran interés por la técnica constructiva, se han conservado parte de la carga, del aparejo del buque y del equipamiento personal de los marineros. Las características del casco indican que se trataba de una nave adaptada para una navegación mixta, ya fuera en mar abierto ó en aguas próximas a la costa ${ }^{14}$.

- Localidad Taglio Nuovo, San Giuseppe di Comacchio (Ferrara): en 1982 se descubrió parte del casco de una nave tardo-romana, de la cual daremos más detalles a continuación.

- Rávena, Parque de Teodorico: en otoño de 1998 se encontró el yacimiento de una barca tardo-romana, a la cual dedicaremos la segunda parte de este artículo.

\section{Toscana}

- Pisa: en diciembre de 1998 se produjo uno de los principales descubrimientos de la arqueología naval del Mediterráneo. Presenta características de gran importancia por el elevado numero de restos materiales, por su excepcional estado de conservación, así como por el contexto más general de carácter portuario en el cual se haya incluido, perteneciente a la época etrusca y romana de la ciudad. Durante los trabajos para la realización de un nuevo centro directivo de la red ferroviaria estatal, cerca de la estación de Pisa- San Rossore, fueron halladas las instalaciones portuarias de la ciudad, en cuyo interior se encontraron al menos dieciséis embarcaciones antiguas, de las que ocho están en curso de excavación (figura 3). Junto a estos, también salieron a la luz los restos de las estructuras portuarias, así como gran cantidad de materiales pertenecientes a la carga, al aparejo de las naves, al equipamiento de a bordo, así como objetos personales de los marineros. Entre estos yacimientos se encuentra uno completamente destruido datado en los primeros decenios del s. II a.C., de cuya carga formaban parte también diversas especies de animales, entre ellos tres caballos y un león. En contraposi- 
ción, por su excepcional estado de conservación, se encuentran los restos de una nave a remo datada en la primera etapa imperial. Bastante singular es otra estructura muy robusta perteneciente a otra nave, situada en posición completamente invertida, de cronología tardo-antigua. Un caso particular es también una embarcación de dimensiones alargada y estrecha, datada en los primeros decenios del s. II d.C. y destinada probablemente a la navegación fluvial y lagunar.

La situación de la investigación permite adscribir el puerto a un arco cronológico de cerca de unos mil años, desde el s. $\vee$ a. C., al s. $\vee$ d.C. Los restos estudiados se datan entre los s. III y II a.C. y el s. V d. C. ${ }^{15}$ Pero los trabajos están en fase de estudio y reservarán, con seguridad, muchos nuevos descubrimientos.

\section{Lazio}

- Fiumicino (Roma): en 1958 los trabajos de reconstrucción del aeropuerto intercontinental "Leonardo Da Vinci" de Fiumicino, cerca de Roma, permitieron durante la investigación arqueológica, el hallazgo de la zona portuaria ordenada construir por el emperador Claudio. Entre los años 1958 y 1965 se recuperaron cinco naves así como fragmentos de otras (figura 4) que actualmente se hayan expuestos en el Museo de las Naves Romanas de Fiumicino. Se trata de cuatro embarcaciones onerarias y de una barca de pesca. Ésta última presenta la singular característica de tener instalada en el centro del casco una "caja-vivero"con el fondo horadado, que permitía mantener el pescado vivo hasta la llegada a puerto, gracias a la renovación del agua del mar que entraba directamente del exterior a través de los agujeros. La datación de estos yacimientos es controvertida; la barca de pesca pertenecería al siglo II d.C., mientras las onerarias a fines del s. II y el siglo IV d. C. Sin embargo, hay que destacar que debido a las características constructivas de dos de ellos (Fiumicino I y 2) podrían ser fechados más tardíamente, entre los siglos IV y $\vee$ d. C. ${ }^{16}$.

\section{Campania}

- Litoral de Sinuessa (Caserta): en 1988, como consecuencia de una violenta marejada, se encontraron en la playa restos de una embarcación de cronología romana; siendo probable que estos no se hallaran in situ, es decir, que fuese un yacimiento secundario al ser dichos restos arrastrados a la playa 17.

Sicilia

- Pantano Longarini (Siracusa): en 1965, durante los trabajos de dragado de un área pantanosa, ocupada en otra época por el mar, fueron encontrados y en gran parte destruidos los restos de una gran nave bizantina fechada entre los siglos VI y VII d.C., conservados en principio en buen estado. Este yaci-

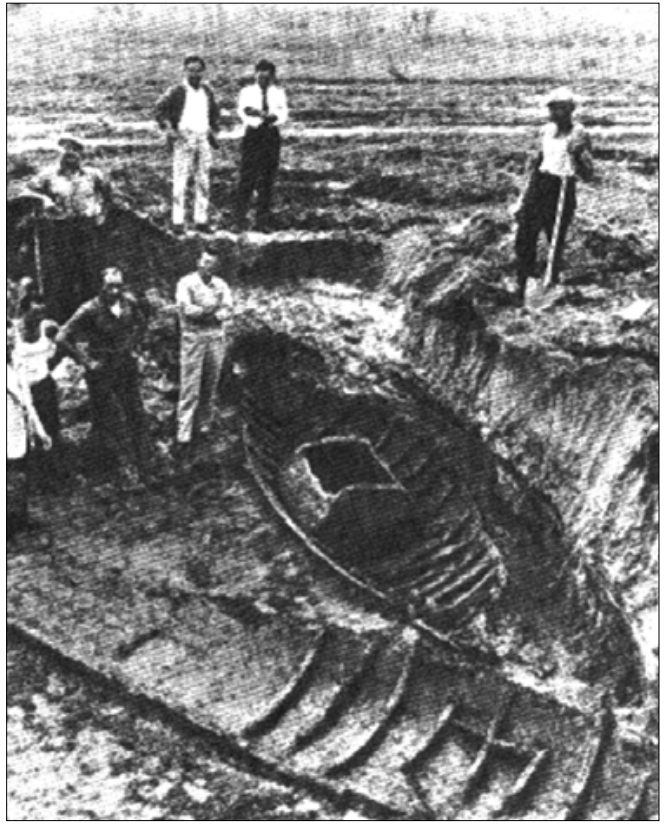

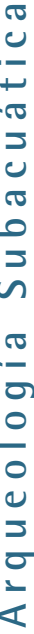

miento posee una particular importancia por la técnica constructiva del casco, a la que haremos referencia más adelante.

\section{Cerdeña}

- Olbia: se trata de un reciente y extraordinario descubrimiento, ocurrido en el área portuaria antigua y medieval. Durante la realización de una obra pública para la construcción de un túnel en una carretera cerca del puerto en el verano de 1999, fueron descubiertos ocho barcos, de los cuales tres eran de cronología tardo-romana y cinco medieval. En el caso de los primeros, se puede proponer la hipótesis de que el hundimiento se debió a una única circunstancia, quizás relacionado con un importante acontecimiento bélico o con un desastroso incendio producido en el transcurso del s. $V$ d.C. Es decir, una situación distinta de la producida en otros contextos portuarios como en el caso de Parque Julio Verne en Marsella o el de Pisa. Las tareas de excavación se hallan todavía en proceso de realización y se estima que al menos otros cinco barcos de época romana se encuentran sepultados en el fango 18.

A continuación de esta presentación general, examinaremos más detalladamente el caso del pecio de Rávena.

\section{El pecio de Rávena}

Con motivo de los trabajos de excavación para la realización del Parque Teodorico en Rávena, el 6 de noviembre de 1998 fueron identificados los restos de una barca tardo-romana, a unos $200 \mathrm{~m}$ al norte del Mausoleo de Teodorico. El pecio se hallaba bajo la arena a unos $8.50 \mathrm{~m}$ de profundidad bajo el actual nivel del suelo, en una zona correspondiente a la anti-
4. Fiumicino: la barca de pesca Gianfrotta-Pomey 198I). durante su excavación (de 
0
0
$+\pi$
2
0
0
0
2
0
0
0
0
0
0
0
2
2
0
1
$<$

5. Rávena, Parque de Teodorico: el pecio libre de sedimentos en el interior (fotografía del autor por cortesía de la Soprintendenza Archeologica dell'Emilia Romagna).

6. Fase final de la excavación; construcción del doble molde y de la estructura de sujeción. gua línea de playa romana y tardo-romana. La intervención arqueológica se inició en diciembre de 1998 y se concluyó en febrero de 1999 con la recuperación de los restos materiales. Los trabajos, dirigidos por personal altamente cualificado en el campo de la arqueología y de la conservación, fueron dirigidos por la Soprintendenza Archeologica de la región de Emilia Romagna, con la colaboración del Instituto Centrale per il Restauro 19

La intervención se inició con la excavación del paquete estratigráfico del interior del casco (figura 5) y posteriormente de los terrenos sobre el que yacía, ofreciendo la posibilidad de ver bien la parte externa y el fondo del casco. Paralelamente a la excavación se desarrollaron los trabajos de protección del pecio, que fue recubierto con un doble molde estanco realizado progresivamente sobre las estructuras conforme eran liberadas de los sedimentos: el primero es un molde de silicona en contacto directo con la madera; el segundo es de resina de vidrio con refuerzos longitudinales y transversales, realizado sobre el primero formando una estructura de gran resistencia mecánica, capaz de garantizar la perfecta estabilidad del pecio.

La excavación del entorno del pecio y el sucesivo recubrimiento con la doble capa se realizaron progresivamente en varias etapas, teniendo en cuenta primeramente el proceso de excavación, después el cubrimiento para su protección y posteriormente el sostenimiento por medio de vigas transversales adaptadas a las distintas partes del casco y apoyadas sobre una estructura metálica de base (figura 6). Ésta estructura ha sido posteriormente completada hasta constituir una "jaula" metálica en cuyo interior ha permanecido todo el sistema pecio-molde; finalmente, todo el complejo fue trasladado al centro de restauración de Comacchio (Ferrara).
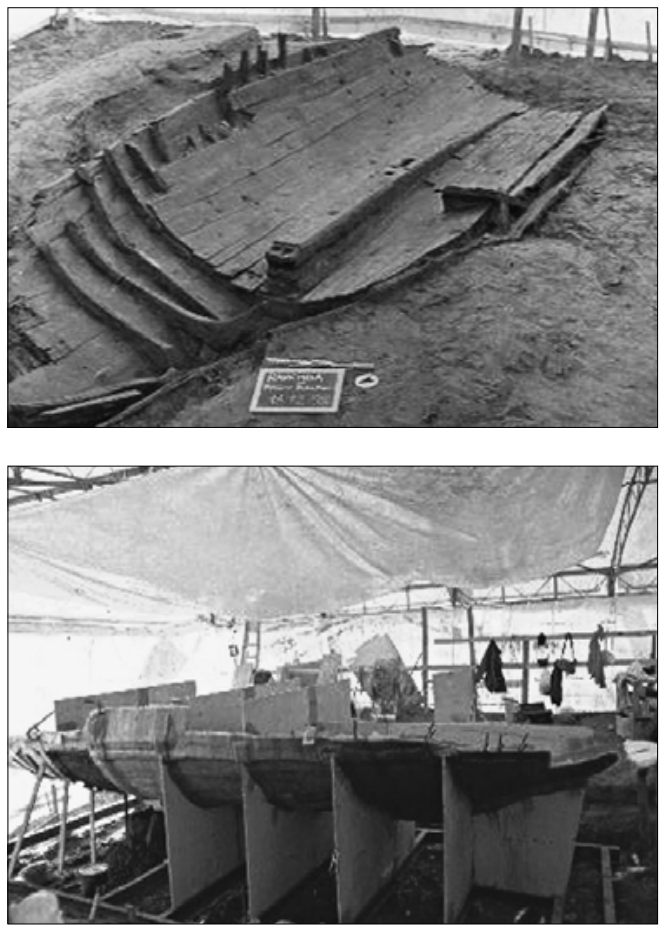

Con este sistema, el pecio ha sido liberado de los sedimentos internos y externos sin sufrir un mínimo movimiento. El sistema adoptado tenía previsto dejar todos los elementos estructurales en su posición originaria, exactamente como se encontraban in situ. La función del doble molde, además de permitir su inmediata protección, es facilitar el tratamiento de consolidación que prevé la impregnación de la madera con la distribución de soluciones líquidas en el interior del molde de goma silicona. Con este objetivo, en el molde se han realizado surcos que permiten la entrada y la recogida de la solución líquida permitiendo de este modo su recirculación.

Durante los trabajos de campo la visibilidad de diversas y particulares estructuras del casco estuvo parcialmente limitada por la presencia de la capa de protección que recubría la tablazón externa. Para evitar dañarlo, se conservó un pequeño estrato de concreción natural que será eliminado cuando se concluyan los trabajos de restauración. Las cuadernas también han permanecido en gran parte oculta por la tablazón interna. Por tanto, las conclusiones que se dan a continuación sólo tienen un carácter preliminar, ya que el estudio detallado de cada una de las estructuras se producirá después de la limpieza definitiva prevista cuando finalice el proceso de consolidación tras la reapertura del molde, proceso que se estima tardará unos cuatro años.

La nave acabó abandonada en la playa; los pocos materiales presentes a bordo, pertenecientes parte a los restos de la carga y parte al equipamiento de a bordo, permiten datar el pecio en el siglo $\vee$ d.C. Entre los materiales destacamos dos lucernas de vidrio de producción siria con sistema de suspensión, una botella de vidrio de tradición oriental de fino espesor con decoración lineal en azul, dos lucernas con factura del vecino oriente, tres vasos de terra sigillata africana, seis calzados de cuero, una olla de hierro, un gancho de hierro y un bastón de madera con un engrosamiento en el extremo.

El yacimiento se caracterizaba por una gran potencia estratigráfica. Numerosos estratos constituidos por arena, turba o por una mezcla de ambos, documentaron el paso de un contexto costero-marino a un medio aéreo, a través de la alternancia de fases de corriente de agua dulce y de ambiente lagunar.

El pecio posee una longitud máxima de 7,22 m y una anchura máxima de 2,75. El costado derecho, que es el mejor conservado, alcanza hasta la altura del la cinta. La popa fue parcialmente alcanzada por la excavadora mecánica que intervino en el yacimiento (posiblemente en un metro aproximadamente), mientras que la proa, de la que se conserva gran parte de la roda, ha sufrido un proceso de hundimiento e inclinación hacia la derecha. En conjunto, el casco está bien conservado, a pesar de presentar cierta deformación debido a las condiciones del yacimiento y al proceso de cubrimiento con arena, que han determinado un alargamiento en sentido longitudinal. 
Al objeto de recuperar parte de la estructura del casco destruida, se llevaron a cabo diversas tareas de reconocimiento del área en la que se había descargado la arena previamente a la intervención arqueológica. Esta zona, formada por diversos montículos de arena (correspondiente cada uno a la descarga de cada camión), era muy amplia y los depósitos fueron removidos varias veces. No obstante, no fue posible identificar una verdadera área de concentración de materiales correspondiente a las descargas que debían contener los restos de la nave. Sin embargo, se encontraron diversos fragmentos de la tablazón, de las cuadernas y un fragmento del codaste con una longitud de alrededor de $70 \mathrm{~cm}$.

En una primera observación sobre el terreno, se pudo determinar que las maderas principalmente utilizadas son encina para la quilla y para las cuadernas, conífera para el resto del casco, madera de abeto para la tablazón exterior e interior y pino para la sobrequilla.

La sobrequilla, de una longitud de 4,50 m se encontraba en una posición ligeramente elevada de proa, lo cual ha permitido investigar la parte más baja del casco y la remoción de la capa de arena que rellenaba el espacio interior entre la tablazón externa y la interna. El levantamiento, producido por la entrada de los detritus, venía determinado por el hecho de que la sobrequilla no se hallaba fijada a los maderos, sino que se encontraba encastrada sobre estos por medio de los nichos abiertos expresamente en la parte inferior, manteniéndose en posición mediante la presión de puntales verticales (figura 7).

La carlinga del palo mayor está realizada directamente en la sobrequilla, ligeramente descentrada hacia proa (alrededor de $50 \mathrm{~cm}$.). El acoplamiento de la base del palo presenta la forma característica con el fondo inclinado hacia la parte posterior, funcional para la maniobra de abatimiento del mismo. La fogonadura debía estar realizada en un través que unía los dos flancos, encastrado entre dos cuadernas y apoyado sobre las dos tablas de la tablazón interna a la altura de la cinta. La cavidad transversal delante de la carlinga del mástil servía probablemente para encajar una pequeña tabla que constituía una rudimentaria caja de fogonadura, privada de cierres laterales. Las aperturas cuadradas talladas en la tablazón interna a los lados de la carlinga del palo (al filo de las varengas) 20 y la caja realizada delante de la cavidad transversal servían probablemente para alojar los pies de los puntales que sostenían el través.

La tablazón interna ocupa la parte central del casco, correspondiente al desarrollo de la sobrequilla, y está unido a las cuadernas con clavos de hierro. En este tramo el casco era abierto, detectándose la presencia de un puente a proa y otro a popa por los nichos existentes en la cinta, en los cuales debían ensamblarse los baos en forma de tabla.

A los dos lados de la sobrequilla se encontraban dos filas de tablas desmontables, apoyadas directamente sobre los maderos, que constituían el enjaretado; de
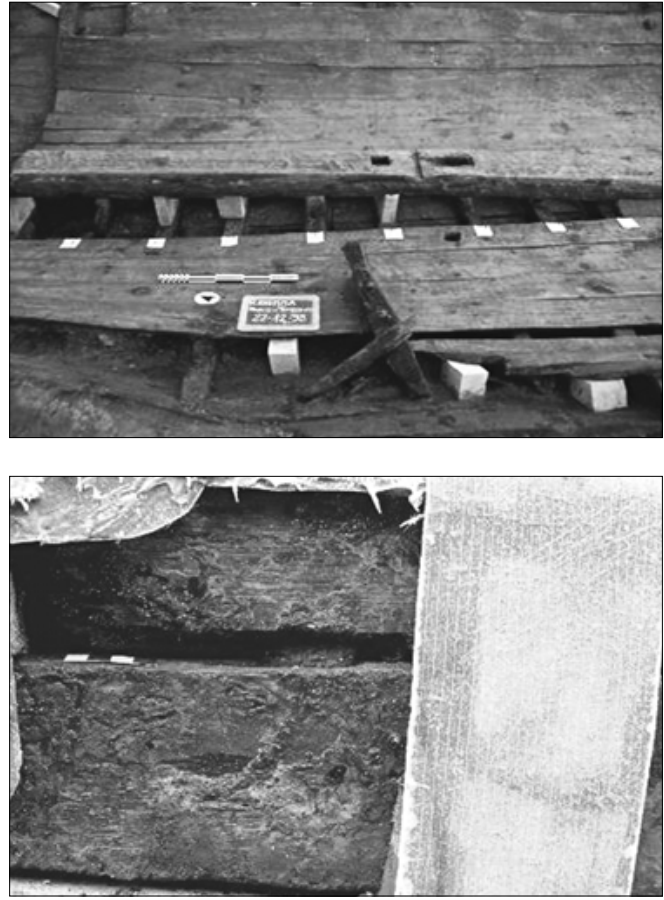
con la sobrequilla liberada de los sedimentos que lo mantenían en posición vertical.

8. Cara externa; detalle de una espiga encastrada en la mortaja, no bloqueada por clavija. este modo permitían alcanzar la sentina para la limpieza periódica de los orificios de achique a los lados de la quilla.

De la estructura se conservan 18 cuadernas y la huella de otra a proa, compuestas de varengas y ligazones. Algunos maderos (varengas) se encuentran fijados directamente a la quilla con un clavo de hierro.

Las maderas de la tablazón externa están ensambladas a las cuadernas con cabillas de madera y con clavos de hierro. Asimismo, se ha constatado la presencia de uniones a espiga al menos hacia la altura de la parte central del pantoque. Las espigas están muy distanciadas unas de otras (cada $80 \mathrm{~cm}$., lo que ha sido posible documentar a través de la costura), están alojadas en mortajas más alargadas que aquellos y se hallan sueltas, sin estar bloqueadas por las cabillas (figura 8). Las tablas, a pesar de estar ensambladas entre ellas, no poseen un blocaje en sentido vertical, teniendo asimismo, un notable juego en sentido longitudinal. La tablazón de esta barca, por tanto, no era sostenible por si misma, a diferencia de aquellos cascos clásicos greco-romanos construidos según el sistema denominado "unión a espiga y mortaja" 21. La solidez del casco estaba completamente asegurada por el esqueleto, convirtiéndose en la verdadera estructura de sustentación (como atestigua también el blocaje de algunas varengas a la quilla por medio de clavos). La utilización esporádica de las espigas debía servir para guiar la colocación de la tablazón durante la construcción, apoyando las tablas al menos hasta el nivel del pantoque, siempre en el límite de cuanto se ha podido revelar hasta ahora.

Este pecio testimonia los últimos éxitos en el siglo $\mathrm{V}$ d.C. del sistema constructivo denominado "sobre tablazón" o "sobre forro", que permitía el progresivo montaje de partes de la tablazón antes de insertar las 
cuadernas. Sí el procedimiento constructivo aparece en gran parte ligado a este sistema clásico, la importancia conferida al esqueleto como principal estructura de sujeción del casco es testigo de una evolución hacia una nueva concepción estructural, que ya puede considerarse enteramente de "construcción sobre cuadernas". Se puede precisar que la concepción del casco y puede que también el procedimiento constructivo, son de tipo "mixto".

Actualmente es posible seguir bastante bien esta evolución en el ámbito mediterráneo, proceso que no se produjo de improviso, sino que se desarrolló en el transcurso de algunos siglos ${ }^{22}$. La fase de transición se inicia con el espaciamiento de la "unión a espiga y mortaja" y la pérdida de regularidad en la disposición ${ }^{23}$, a la cual corresponde un rol más activo del esqueleto. Esta primera fase está bien documentada en el pecio del s. IV d.C. de Yassi Ada II (Turquía) ${ }^{24}$, así como, en la parte norte del Adriático, en el de San Giuseppe di Comacchio (Ferrara), que podría datarse en el s. IV d.C. ${ }^{25}$.

Un nuevo examen realizado recientemente en el pecio I de Fiumicino (Roma) ha permitido detectar la presencia de "uniones a espiga y mortaja" muy distanciadas entre sí, algunas de las cuales contienen espigas libres, no bloqueados por cabillas. Las características técnicas de la estructura del casco de la nave, que junto a los materiales hallados en asociación con el pecio, indicarían una cronología entre los s. IV y $\vee \mathrm{d}$. C. ${ }^{26}$, asumen un papel muy significativo en el estudio de la evolución de los sistemas constructivos.

El pecio de Yassi Ada I (Turquía), datado en el s. VII d. C. ${ }^{27}$, se halla en una fase más avanzada de esta transición: el fondo del casco hasta el pantoque se encuentra todavía construido ensamblando las tablas con el método de "unión a espiga y mortaja", que sin embargo no tienen ninguna función de sujeción, encontrándose en una posición muy distanciada y sin cabillas de blocaje ${ }^{28}$, mientras que las partes altas de los flancos estaban realizadas "sobre cuadernas". Un procedimiento similar ha sido constatado en el pecio del Pantano de Longarini (Sicilia), perteneciente a una gran nave mercantil de los siglos VI o VII d.C. ${ }^{29}$.

El pecio de Saint-Gervais II (For-sur-Mer, Francia) ${ }^{30}$, perteneciente al siglo VII d.C., documenta la última etapa de esta evolución, representada por un casco cuyo fondo parece haber sido construido completamente "sobre cuadernas". Sólo pocas uniones a espiga, muy distanciadas entre sí y carentes de cabillas de bloqueo ${ }^{31}$, testimonian todavía la persistencia del viejo sistema de ensamblaje. En el siglo VII las espigas no bloqueadas por las cabillas aparecen siempre en las maderas de la tablazón que constituyen los pocos restos del pecio de Dor D, Laguna di Tantura (Israel) ${ }^{32}$

El pecio de Rávena prueba que la fase avanzada de esta evolución era ya una realidad en las pequeñas embarcaciones datadas en torno a la primera mitad o a mediados del s.V d.C. 33 .

Descubrimientos recientes sin embargo, documentan que en el área mediterránea el proceso de transición se haya casi finalizado para las pequeñas embarcaciones ya en el s. VI d.C., si bien no de un modo generalizado, como atestigua el pecio A de la Laguna de Tantura perteneciente a una embarcación que medía originariamente $12 \mathrm{~m}$ de longitud y alrededor de $4 \mathrm{~m}$ de ancho, construida completamente "sobre cuadernas" 34. Al menos desde un período comprendido entre la mitad del s. VIII d.C. y la mitad del s. IX d.C. (siempre basándose en los datos disponibles en la actualidad) el sistema "sobre cuadernas" ó "sobre el esqueleto" era utilizado en el ámbito del Mediterráneo para la construcción de naves de grandes dimensiones, como queda probado en el pecio B de la Laguna de Tantura ${ }^{35}$

Como esbozo, la tablazón externa conserva extensas porciones de la capa protectora de pez que lo recubría ${ }^{36}$, además de una banda de color blanco que se extiende en sentido longitudinal por debajo de la cinta.

En base a la reconstrucción gráfica de la línea del casco ${ }^{37}$, se puede plantear la hipótesis de que la barca de Rávena tuviera originariamente una longitud de unos $9 \mathrm{~m}$ aproximadamente, una anchura de alrededor $3.10 \mathrm{~m}$, y un desplazamiento de 4.85 toneladas. El casco presentaba líneas bien marcadas, ahusándose hacia los extremos, mientras que en la parte central tenía un fondo más bien plano; la quilla sobresalía de la tablazón externa unos $8-10 \mathrm{~cm}$. de media, constituyendo un marcado plano de deriva; la proa era un poco más alta que la popa. Se trataría de un casco de gran calidad náutica, adaptado tanto para la navegación en el mar como en aguas interiores, en plena correspondencia con el tipo de navegación que debía desarrollarse en el área de Rávena, ya que existía una estrecha relación entre la navegación costera y la navegación intralagunar.

Finalmente, hay que destacar que la calidad constructiva de esta embarcación en conjunto es somera y con un acabado un poco acelerado, ya que diversos elementos se hallan mal terminados y montados con cierto descuido, contrastando sin embargo con las líneas afiladas y elegantes del casco. 
BASCH, L., 1986, Note sur le calafatage: la chose et le mot. Archaeonautica, 6, pp. $187-198$.

BASS, G. F. \& VAN DOORNINCK, F. H., 1978, An I Ith century shipwreck at Serçe Liman, Turkey. International Journal of Nautical Archaeology, 7, pp. 119-132.

BASS, G. F. \& VAN DOORNINCK, F. H., 1982, Yassi Ada, vol. I. A seventh-century byzantine shipwreck, College Station, Texas.

BELTRAME, C., 1996, La sutilis navis del Lido di Venezia. Nuova testimonianza dell'antica tecnica cantieristica a cucitura nell'Alto Adriatico. Navalia. Archeologia e Storia, F. Ciciliot (Ed.), Savona 1996, pp. 31-53.

BELTRAME, C., 1996/1997, Sutiles naves e navigazione per acque interne in età romana. Padusa, XXX|I/XXXIII, n.s., pp. I37- 146.

BERTACCHI, L. - P., 1988, L'imbarcazione romana di Monfalcone, Udine.

BERTI, F., 1986, Rinvenimenti di archeologia fluviale ed endolagunare nel Delta ferrarese. Bollettino d'Arte, n. 37-38, Supplemento, Archeologia Subacquea 3, Roma, pp. 19-38.

BOETTO, G., 2000, New technological and historical observations on the Fiumicino I wreck from Portus Claudius (Fiumicino, Rome). Down the river to the sea. Proceedings of the Eighth International Symposium on Boat and Ship Archaeology, Gdánsk 1997, J. Litwin (Ed.), Gdánsk 2000, pp. 99-102.

BONINO $1978=$ M. BONINO, Archeologia e tradizione navale tra la Romagna e il Po, Ravenna 1978.

BONINO, M., 1983/1984, L'imbarcazione di Taglio Nuovo (S. Giuseppe di Comacchio). Musei Ferraresi, I3/I4, pp. 55-58.

BONINO, M., 1991, Archeologia navale. Storia di Ravenna, II, I. Dall'età bizantina all'età ottoniana, A. Carile (Ed.), Venezia, pp. 27 53.

BONINO, M., 2000, Una barca medievale rinvenuta a Porto Fuori (Ravenna). Archeologia delle Acque, 4 (luglio/dicembre), pp. 48-53.

BRUNI, S., 2000, II porto urbano di Pisae e i relitti del complesso ferroviario di "Pisa-San Rossore". Le navi antiche di Pisa, pp. 21 -79.

Contarina 1898 = Sulla scoperta di due barche antiche nel territorio del Comune di Contarina in provincia di Rovigo, nel gennaio 1898. Relazione della Commissione eletta dalla R. Deputazione Veneta di Storia Patria, Venezia 1900.

DELL'AMICO, P., 1998, Appunti sui cambiamenti intervenuti nelle costruzioni navali mediterranee dall'antichità ai giorni nostri. Navi di legno. Evoluzione tecnica e sviluppo della cantieristica nel Mediterraneo dal XVI secolo a oggi. Atti del Convegno Internazionale, Grado, 21-25 maggio 1997, M. Marzari (Ed.), Trieste, pp. 17-26.

D'AGOSTINO, M., MEDAS, S., en prensa, I relitti dell'isola di S. Marco in Boccalama, Venezia. Rapporto preliminare. Atti del II Convegno Nazionale di Archeologia Subacquea, Castiglioncello 7-9 settembre 2001 .
D'AGOSTINO, M., MEDAS, S., en prensa, Lo scavo dei relitti di San Marco in Boccalama, Laguna di Venezia. Notizia preliminare. Archeologia delle Acque, 6 (luglio/dicembre 200I).

D'ORIANO, R., 2000, I relitti del porto romano di Olbia: notizia preliminare. Alétes. Miscellanea per i settant'anni di Roberto Caprara, Massafra, p. 231-237.

FOERSTER, F., 1990, La protección de la madera de las naves marítimas en la Antigüedad. Le commerce maritime romain en Méditerranée occidentale $=$ PACT, 27, pp. 183-187.

FORTUNA MARIS. La nave romana di Comacchio, F. Berti (Ed.), Bologna 1990.

GADDI, D., 1999, Grado e il sistema portuale di Aquileia. AA.WV., Operazione lulia Felix. Dal mare al museo, Mariano del Friuli (GO), p. 17-26.

GIANFROTTA, P. \& POMEY, P., 198I, Archeologia subacquea. Storia, tecniche, scoperte e relitti, Milano.

JÉZÉGOU, M.-P., 1985, Eléments de construction sur couples observés sur une épave du haut Moyen Age découverte à Fos-surMer. VI Congreso Internacional de Arqueologia Submarina, Cartagena 1982, Madrid, pp. 35I-356.

JONCHERAY, J.-P., 1975, Une épave du Bas Empire: Dramont F. Cahiers d'Archéeologie Subaquatique, 4, pp. 91-140.

KAHANOV, Y., 2000, The Tantura B Shipwreck. Tantura Lagoon, Israel. Preliminary hull construction report. Down the river to the sea. Proceedings of the Eighth International Symposium on Boat and Ship Archaeology, Gdánsk 1997, J. Litwin (Ed.), Gdánsk 2000, pp. |5|-|54.

KAHANOV, Y. \& ROYAL, J. G., 1996, The 1995 INA/CMS Tantura A Byzantine Shipwreck Excavation - Hull Construction Report. C.M.S. News, 23 (December), pp. 2I-23.

KAHANOV, Y. \& ROYAL, J. G., en prensa, Analysis of Hull Remains of the Dor D Vassel, Tantura Lagoon, Israel. International Journal of Nautical Archaeology.

LE NAVI ANTICHE DI PISA. Ad un anno dall'inizio delle ricerche, S. Bruni (Ed.), Firenze 2000.

MAIOLI, M. G., 1986, Cervia (Ravenna). Relitto bizantino o altomedievale. Bollettino d'Arte, n. 37-38, Supplemento, Archeologia Subacquea 3, Roma, pp. 14-16.

MAIOLI, M. G., MEDAS, S., 200I, II relitto tardo-romano del Parco di Teodorico, Ravenna: dallo scavo al recupero. NAVIS. Rassegna di studi di archeologia, etnologia e storia navale, M. Marzari (Ed.), 2 , Sottomarina (VE), pp. I05- 135.

MARSDEN, P., 1994, Ships of the Port of London. First to eleventh centuryes AD, London.

MEDAS, S., 2000, Archeologia subacquea, archeologia delle acque e archeologia navale nel medio e alto Adriatico italiano, tra passato, presente e futuro. Ravenna Studi e Ricerche, VII/I, pp. 65-84. 
MEDAS, S., 1999, II relitto tardo-romano del Parco di Teodorico a Ravenna. Nota preliminare. Archeologia delle Acque, 2 (luglio/dicembre), pp. 135-138.

MEDAS, S., en prensa, Shipbuilding preliminary notes on the Late-Roman wreck recently discovered in the Parco di Teodorico in Ravenna, Italy. Boats, ships and shipyard. IX International Symposium on Boat and Ship Archaeology, Venice, 4-8th December 2000, Proceedings.

MELILLO FAENZA, L. \& PICCIOLI, C., 1992, Resti di imbarcazione a Sinuessa. La conservazione e il restauro. II trasporto commerciale marittimo nell'antichità, A. Fratta (Ed.), Genova, pp. 89-92.

POMEY, P., 1988, Principes et methodes de construction en architecture navale antique. Navires et comerce de la Méditerranée antique. Hommages à J. Rougè = Cahiers d'Histoire, 33, pp. 397-4I2.

POMEY, P., 1994, Shell Conception and Skeleton Process in Ancient Mediterranean Shipbuilding. Crossroads in Ancient Shipbuilding. Proceedings of the Sixth International Symposium on Boat and Ship Archaeology, Roskilde 1991, ISBSA 6, Oxford, pp. 125-130.

POMEY, P., 1995, Les épaves grecques et romaines de la Place Jules-Verne à Marseille. Comptes Rendus de l'Académie des Inscriptions \& Belles Lettres, avril-juin 1995, pp. 459-484.

POMEY, P., 1997, Les navires, in La Navigation dans l'Antiquité, sous la direction de Patrice Pomey, Aix-en-Provence, pp. 60-101.

POMEY, P., 1998, Conception et réalisation des navires dans l'Antiquité méditerranéenne. Concevoir et construire les navires. De la trière au picoteux, sous la direction de Eric Rieth, Ramonville, pp. 49-72.
RICCARDI, E., 2000, I relitti del tunnel di Olbia. L'Archeologo Subacqueo, 18 (settembre-dicembre), p. 2.

ROYAL, J. G. \& KAHANOV, Y., 2000, An Arab-Period merchant vassel at Tantura Lagoon, Israel. International Journal of Nautical Archaeology, 29, pp. 151-153.

SCRINARI, V. S. M., 1979, Le navi del porto di Claudio, Roma.

THROCKMORTON, P. \& J., 1973, The roman wreck at Pantano Longarini. International Journal of Nautical Archaeology, 2, pp. 243-266.

TORTORICI, E., 1997, Archeologia subacquea e trasformazioni geomorfologiche del territorio: il caso della Laguna di Grado. Atti del Convegno Nazionale di Archeologia Subacquea, A.l.A.Sub, Anzio, 3031 maggio e $1^{\circ}$ giugno 1996, Bari 1997, pp. 315-325.

TORTORICI 1998 = E. Tortorici, Lo scavo subacqueo. Archeologia Subacquea. Come opera l'archeologo sott'acqua. Storie dalle acque. VIII Ciclo di lezioni sulla ricerca applicata in archeologia, Certosa di Pontignano (Siena), 9-15 dicembre 1996, Firenze 1998, pp. 29-62.

UCELLI, G., 1949, Le navi di Nemi, Roma.

VAN DOORNINCK, F., 1974, Bisanzio, signora del mare (330-64I). Navi e Civiltà. Archeologia marina, G. F. Bass (Ed.), Milano, pp. 133-158.

VAN DOORNINCK, F. H., 1976, The 4th century wreck at Yassi Ada. An interim report on the hull. International Journal of Nautical Archaeology, 5, pp. ||5-|3|.

WACHSMANN, S. \& KAHANOV, Y., 1997, Shipwreck Fall: the 1995 INA/CMS Joint Expedition to Tantura Lagoon, Israel. INA Quarterly, 24, I, spring, pp. 3-18.

\section{Notas}

I. Recordamos que entre otros, el reciente descubrimiento de Place Jules-Verne en Marsella (Pomey 1995).

2. Para el caso de la laguna de Grado véase Tortorici 1997.

3. Véase a tal propósito, las oportunas consideraciones de $M$. D'Agostino en la introducción de Archeologia delle Acque, 2 (julio/diciembre 1999) p.7, y Medas 2000, pp. 65-66.

4. Véase la presentación de L. Fozzati en Archeologia delle Acque, I (enero-julio 1999), pp. 4-5; editorial y artículo de J. Coles (Archeologia delle Acque: oportunidad y perspectivas futuras) en la misma revista, pp. 6-16

5. Véase Gianfrotta-Pomey 1981, pp. 18-39, 230; Tortorici 1998, pp. 29-36. En referencia a los pecios de Nemi (Ucelli 1940). Hace poco (desde junio hasta octubre del 200I) han sido excavados dos grandes peciso medievales de la primera mitad del siglo XIV en San Marco de Boccalama, en la laguna de Venecia. El trabajo, técnicamente muy complejo, ha contemplado la excavación estratigráfica subacuática de los pecios y, sucesivamente, la puesta en seco de toda el área para ejecutar el trazado fotogramétrico (D'Agostino-Medas en prensa, a y b).

6. Contarina 1898; Bonino 1978, pp. 63-64, 84.

7. A pesar del diverso grado de veracidad, no son pocas las referencias orales que circulan relacionadas con restos de embarcaciones que se encontraron durante los distintos trabajos de excavación en el territorio.

Pertenecen asimismo a la lista de restos las embarcaciones monóxilas encontradas en las turberas o en otros contextos terrestres, sobretodo en Italia septentrional, para los cuales se hace necesario un contextualización ambiental y arqueológica específico. Sin embargo, en nuestro estudio examinaremos sólo los restos materiales relacionados, directa o indirectamente, con la zona costera y la zona intermareal.

8. Sin entrar en detalles, hay que recordar que se localizaron en las playas a lo largo del litoral diversos restos de embarcaciones, transportados desde el mar hacia la playa por marejadas especialmente violentas. Éste es el caso de los restos de una embarcación romana hallada entre los años 1993 y 1994 en la playa de los Alberoni, en la isla de Lido, Venecia (Beltrame 1996). También podría pertenecer a este tipo de contexto (pecio transportado por marejadas hacia la playa) los restos de Sinuessa (infra).

8. Bertacchi 1988.

9. Tortorici 1997, p. 322; Gaddi 1999, p.21.

10. Sobre estos hallazgos véase Beltrame 1996/1997.

1 1. Sobre estos restos del área del delta del Po, en la provincia de Ferrara, véase Bonino 1978, pp. 19-23, 53-55 y Berti 1986, pp. 19-25.

12. Bonino 1978, pp. 40-42; Maioli 1986, pp. I4-I6.

13. Bonino 2000.

14. FORTUNA MARIS.

15. LE NAVI ANTICHE DI PISA.

16. Scrinari 1979. Entre las características técnicas que inducen a pensar en una datación más baja al menos para el pecio de Fiumicino I (s. IV-V d.C.) podemos considerar la considerable distancia que separa las "uniones a espiga" en las tablas de la tablazón y la presencia de algunas espigas libres, no bloqueadas por cabillas (Boetto 2000). 
17. Melillo Faenza-Piccioli 1992

18. D'Oriano 2000; Riccardi 2000.

19. Medas 1999; Maioli-Medas 200 I; Medas (en prensa)

20. Debía tratarse de puntales con el pie tallado en forma de $L$ invertida, es decir con un diente saliente y correspondiente a las dimensiones de las aperturas cuadradas realizadas en la tablazón interior. De este modo, el diente serviría para bloquear el pie dentro de la apertura, mientras que el empuje vertical descargaría sobre la otra mitad del mismo pie, que apoyaba sobre la tablazón en correspondencia con la cuaderna de detrás de la apertura.

21. En los cascos construidos con este sistema era fundamental la regularidad de cada unión y una distancia cercana, debido a que solamente con una fuerte cohesión entre las tablas el casco podía asumir su función de ser sustentable por si mismo. Las espigas distaban generalmente no más de $10 \mathrm{~cm}$. entre ellas, hallándose encastradas a medida (al objeto de evitar el movimiento) y fijadas con cabillas transversales. Las cuadernas poseían fundamentalmente una función de refuerzo, pero no desempeñaban un rol independiente en la solidez estructural del casco: El ensamblaje de las cuadernas con la quilla estaba asegurado principalmente por la unión entre la traca de aparadura y la quilla misma, siempre a través de la tablazón, a pesar de que no faltan ejemplos en los cuales algunos maderos se hallaban fijados a la quilla con pernos de metal. Cuando está presente, la fijación directa de las varengas a la quilla indica no sólo la voluntad de reforzar la estructura de un casco con tablazón sustentable por si misma, sino también una primera señal de cambio en la concepción estructural del casco mismo, en cuyo interior el papel del esqueleto comienza a asumir mayor importancia como estructura de sustentación. Véase acerca de estos argumentos Gianfrotta-Pomey 1981, pp. 240-247, 260-266; Pomey 1988; 1994; 1997, p.p. 93-100; Dell'Amico 1998.

22. Sobre el proceso que permitió la evolución del sistema constructivo "sobre tablazón" o "sobre forro" al denominado "sobre cuadernas", en el que se incluye la distinción entre la concepción y la construcción del casco, son fundamentales los trabajos de investigación de Patrice Pomey (1988; 1977, pp. 97-100; 1988).

23. En general, se ha constatado un cuidado siempre menor al realizar el ensamblaje de las tablas: la distancia entre las mortajas se convierten siempre en irregulares, disminuyendo también la precisión de los encastres, con mortajas mucho más alargadas que las espigas.

24. van Doorninck 1974, pp. 136-138; van Doorninck 1976. La distancia media entre las espigas, desde sus partes centrales, es de $25 \mathrm{~cm}$. (oscila entre un mínimo de $15 \mathrm{~cm}$. y un máximo de $30 \mathrm{~cm}$.).

También el pecio de Dramont F (s. IV d.C.) es testimonio del inicio de este proceso evolutivo: la utilización de "unión a espiga y mortaja" comienza a disminuir y a realizarse con menor cuidado (las espigas son más pequeñas que las mortajas y algunas de éstas carecen de espigas) mientras que paralelamente, se nota el ensamblaje directo de al menos una varenga a la quilla (Joncheray 1975).

25. Bonino 1983/1984; Bonino 1991, p. 31. Se desconoce el contexto original de este pecio, cuyo hallazgo y posterior destrucción se produjo de manera fortuita en el año 1982 durante los trabajos de una excavadora mecánica. La distancia de las espigas, de centro a centro, varía desde los 18,5 a los $3,45 \mathrm{~cm}$ Proponemos la datación de este pecio en el s. IV d.C., basándonos en la unión "a espiga y mortaja" contrastadas en los pecios de Dramont F, de Fiumicino I y de Yasi Ada II, pero advertimos que la referencia exclusiva del proceso constructivo nos aporta solamente un valor cronológico relativo.

26. Boetto 2000. La distancia entre las cabillas que fijan las espigas es irregular, una media de $41,5 \mathrm{~cm}$., pero con una variación comprendida entre $17,5 \mathrm{~cm}$ de mínima y $76 \mathrm{~cm}$. de máxima.

27. van Doorninck 1974, pp. 139-|43; Bass-van Doorninck 1982.

28. La distancia entre las espigas, de centro a centro, varía de 30 a $40 \mathrm{~cm}$. a popa, hasta un máximo de alrededor $90 \mathrm{~cm}$. en la parte central del casco.

29. Throckmorton 1973; van Doornick 1974, pp. 143-|44. Las espigas, carentes de cabillas de bloqueo y utilizadas sólo para ensamblar las tablas del casco hasta la altura de la línea de flotación, mantienen entre ellas una distancia de aproximadamente un metro.

El pecio "cosido" de Cervia (Rávena), datado en un período comprendido entre los siglos IV y VI d. C., también podría ser incluido en esta fase del proceso evolutivo: las tablas de la tablazón, están efectivamente ligadas unas a otras solamente en el fondo del casco, mientras que a partir del pantoque parece que han sido directamente fijadas a las cuadernas con cabillas de madera (Bonino 1991, pp.29-31).

30. Jézégou 1985; Pomey 1988, pp. 410-4II.

31. El espacio entre las uniones llega a superar I m.

32. Kahanov-Royal (en prensa). El intervalo medio entre las uniones está comprendido entre los 26 y los $28 \mathrm{~cm}$. (sólo existen pocas excepciones que superen este intervalo, hasta alcanzar cerca de $62 \mathrm{~cm}$.)

33. Por las razones expuestas, solamente falta todavía verificar si la presencia de las espigas concierne a toda la tablazón externa, también por encima del pantoque. En este caso, en efecto, el pecio de Rávena atestiguaría una fase evolutiva inmediatamente anterior a los yacimientos de Yassi Ada I y del Pantano de Longarini, en los cuales la presencia de las espigas se detecta solamente en las partes de la base de la tablazón.

34. Kahanov-Royal 1996; Wachsmann-Kahanov 1997. Este pecio, por tanto, permite aumentar notablemente la cronología en la cual se habrían documentado los primeros cascos de construcción "sobre cuadernas" en el Mediterráneo, cuyo primer testimonio era representado hasta hace pocos años por el pecio de Serçe Liman (Turquía), del s. XI d. C. (Bass-van Doorninck 1978; Pomey 1988, p. 4I I). Debemos recordar que en el norte de Europa existía ya desde hacía mucho tiempo una tradición constructiva "sobre cuadernas", como documentan los pecios de Blackfriars y de New Guy's House en Londres, datados en el s. II d.C. (Marsden 1944, pp. 33- 104).

35. Kahanov 2000. Otro pecio de la Laguna de Tantura, datado en el s. VIII-IX d.C., parece documentar una estructura con la técnica de construcción "sobre cuadernas", no habiéndonse individualizado hasta el momento trazas de unión "a espiga y mortaja". Se trata generalmente de un dato preliminar, derivado del estudio de un área limitada del yacimiento (RoyalKahanov 2000). Los pecios investigados durante los últimos cinco años en la Laguna de Tantura son muy importantes para ver la transición del sistema constructivo "con tablazón de sustentación" hacia el sistema "a esqueleto de sustentación", así como para establecer la cronología de este proceso, que se confirma complejo y difuminado en el tiempo, aplicándose primeramente en los cascos de pequeñas dimensiones. En el contexto del proceso evolutivo de la construcción naval, resulta interesante otro reciente descubrimiento, el del pecio de la "nave D" de Pisa, todavía en fase de excavación, datado en época tardía (fase avanzada del s.V d.C. o posterior), cuya tablazón parece fijado a las cuadernas sólo con clavos de hierro (Bruni 2000, pp. 48-51).

Por tanto, hay que decir que el panorama de la documentación se está enriqueciendo y que se producirán ciertamente nuevos e importantes descubrimientos.

36. Sobre los sistemas utilizados para proteger la tablazón externa (Foester 1990). No se trata de un calafateado en el sentido propio del término (no habiendo sido identificadas hasta la fecha trazas de estopa en las costuras de los tablones), sino de un capa protectora e impermeabilizante, igual al documentado en otros pecios, como por ejemplo los de Chrétienne C (s. II a.C), Port Vendres I (finales del s. IV- inicios del s. V d.C.), Dramont F (finales del s. IV- inicios del s. $V$ d.C.), Pointe de la Luque B (s. III-IV d.C.), Yassi Ada I y II (respectivamente, s. VII d. C. y s. IV d.C.). Sobre este tema véase Basch 1986.

37. Véase al respecto el trabajo de M. Bonino en Maioli-Medas 2001, pp. 120-122. 